\title{
Theoretical Approach to the Gauge Invariant Linear Response Theories for Ultracold Fermi Gases with Pseudogap
}

\author{
Hao Guo ${ }^{1}$ and Yan $\mathrm{He}^{2}$ \\ ${ }^{1}$ Department of Physics, Southeast University, Nanjing 211189, China \\ ${ }^{2}$ College of Physical Science and Technology, Sichuan University, Chengdu, Sichuan 610064, China
}

Correspondence should be addressed to Yan He; heyan_ctp@scu.edu.cn

Received 22 June 2015; Accepted 2 August 2015

Academic Editor: Artur P. Durajski

Copyright (C) 2015 H. Guo and Y. He. This is an open access article distributed under the Creative Commons Attribution License, which permits unrestricted use, distribution, and reproduction in any medium, provided the original work is properly cited.

\begin{abstract}
Recent experimental progress allows for exploring some important physical quantities of ultracold Fermi gases, such as the compressibility, spin susceptibility, viscosity, optical conductivity, and spin diffusivity. Theoretically, these quantities can be evaluated from suitable linear response theories. For BCS superfluid, it has been found that the gauge invariant linear response theories can be fully consistent with some stringent consistency constraints. When the theory is generalized to stronger than BCS regime, one may meet serious difficulties to satisfy the gauge invariance conditions. In this paper, we try to construct density and spin linear response theories which are formally gauge invariant for a Fermi gas undergoing BCS-Bose-Einstein Condensation (BEC) crossover, especially below the superfluid transition temperature $T_{c}$. We adapt a particular $t$-matrix approach which is close to the $G_{0} G$ formalism to incorporate noncondensed pairing in the normal state. We explicitly show that the fundamental constraints imposed by the Ward identities and Q-limit Ward identity are indeed satisfied.
\end{abstract}

\section{Introduction}

Recently there is a broad literature on the subjects of response functions in superconductors and atomic Fermi gas superfluids [1-11], where interparticle interaction is strong enough such that the classical BCS theory is not adequate. Related experiments include the studies of the thermodynamic response functions and dynamical response [12-17]. Theoretically, linear response theories have been an important tool for studying the transport and dynamic properties of Fermi gases. Hence, it is important to assess the self-consistency of the linear response theories as well as comparing with the experimental results. There must be some general rules that the theory must follow. In [18, 19], several fundamental constraints associated with the conservation laws/Ward identities and the sum rules were addressed. Since the conservation laws are generically related to certain (gauge) symmetry of the theory, then in the brokensymmetry phase or ordered phase it is particularly difficult for many-body theories to satisfy all these constraints. It was also pointed out that the strict weakly interacting BCS mean field theory does pass all these types of testing both below and above $T_{c}$ even when the pairing population is unbalanced
$[18,19]$. In other words, the linear response theories of BCS superfluids can be formulated into a fully gauge invariant theory. In the normal state, the simplest Nozieres-SchmittRink (NSR) is also compatible with these gauge invariance conditions [20].

In this paper, we try to build an ideal linear response theory for strongly correlated superfluids undergoing BCSBEC crossover by a diagrammatic approach such that the fundamental constraints mentioned above can be satisfied. Our selected diagrams bear on those associated with the Goldstone modes due to the symmetry-breaking via the consistent-fluctuation-of-the-order-parameter (CFOP) approach and the conventional contributions, namely, the Maki-Thompson (MT) and Aslamazov-Larkin (AL) diagrams. As a price, we have to adapt a slightly modified $G_{0} G$ formalism to incorporate the pairing fluctuation effect. We emphasize that this approach is purely a theoretical attempt until now. However, it might be a necessary step to fully understand the transport properties of strongly correlated Fermi gases.

The linear response theories must be consistent with several fundamental constraints $[18,19,21]$ imposed by 
the Ward identities and Q-limit Ward identity [22]. It is well known that the Ward identities guarantee the gauge invariance of the theory. However, the Q-limit Ward identity is quite strange to most researchers in the condensed matter community. It in fact leads to the sum rules of compressibility and spin susceptibilities which further build the consistent connection between the single-particle thermodynamics and two-particle correlation functions. For spin response theory the Q-limit Ward identity is only meaningful to polarized Fermi superfluids [19]. In this paper, we focus on the unpolarized Fermi superfluids. The central difficulty of formulating the consistent linear response theory is to maintain the gauge invariance when the pseudogap self-energy is introduced by the pairing fluctuation effect. This is obviously beyond the CFOP approach since the total energy gap is now different from the order parameter.

In the following sections, we first briefly review the CFOP linear response theories in both the density and the spin channels for BCS mean field theory; then we introduce the pairing fluctuation effects via a particular $t$-matrix formalism. We further carry on extra diagrammatic corrections in the two channels and verify that the new theories do maintain the gauge symmetry, respectively. Throughout this paper, we follow the convention $c=\hbar=k_{B}=1$.

\section{BCS Mean Field Theory Approach}

By using the $\sigma$ to denote the spin or pseudospin $\uparrow, \downarrow$, the Hamiltonian for a two-component Fermi gas interacting via the attractive contact interaction $g$ is

$$
\begin{aligned}
H= & \int d^{3} \mathbf{x} \psi_{\sigma}^{\dagger}(\mathbf{x})\left(\frac{\widehat{\mathbf{p}}^{2}}{2 m}-\mu\right) \psi_{\sigma}(\mathbf{x}) \\
& -g \int d^{3} \mathbf{x} \psi_{\uparrow}^{\dagger}(\mathbf{x}) \psi_{\downarrow}^{\dagger}(\mathbf{x}) \psi_{\downarrow}(\mathbf{x}) \psi_{\uparrow}(\mathbf{x}),
\end{aligned}
$$

where $\psi$ and $\psi^{\dagger}$ are the annihilation and creation operators of fermions, $\mu$ is the chemical potential, and $m$ is the fermion mass. There is an implicit summation over the pseudospin indices $\sigma$. The Hamiltonian has a $U(1) \times U(1)$ symmetry [23]

$$
\begin{aligned}
& \psi_{\sigma} \longrightarrow e^{-i \alpha} \psi_{\sigma}, \\
& \psi_{\sigma}^{\dagger} \longrightarrow e^{i \alpha} \psi_{\sigma}^{\dagger} ; \\
& \psi_{\sigma} \longrightarrow e^{-i S_{\sigma} \alpha} \psi_{\sigma}, \\
& \psi_{\sigma}^{\dagger} \longrightarrow e^{i S_{\sigma} \alpha} \psi_{\sigma}^{\dagger},
\end{aligned}
$$

where $S_{\uparrow, \downarrow}= \pm 1$ and $\alpha$ is the phase parameter of those transformations. The first $U(1)$ symmetry is well known for relating to the electromagnetism (EM). If the particle is charged, this symmetry naturally becomes a gauge symmetry. For a charge neutral system, the symmetry is still associated with the mass current conservation. The second symmetry is the spin rotational symmetry which is associated with the spin current conservation. Our linear response theories in the density and spin channels must respect these two symmetries, respectively. The central idea is to "gauge" the $U(1)$ symmetries by introducing two types of weak external fields. In the density channel, it is the weak EM field $A^{\mu}=(\phi, \mathbf{A})$, while in the spin channel it is $A^{\mu} \equiv\left(B_{z}, \mathbf{m}\right)$, where $B_{z}$ is the $z$ component of the magnetic field (assuming $z$ is the axis of spin rotation) and $\mathbf{m}$ is the magnetization.

After taking BCS mean field approximation, the order parameter or superconducting gap function is introduced $\Delta_{\text {sc }}(\mathbf{x})=-g\left\langle\psi_{\uparrow}(\mathbf{x}) \psi_{\downarrow}(\mathbf{x})\right\rangle$. The first $U(1)$ symmetry is spontaneously broken while the second is not. As can be seen in [18], this brings significant difference between the linear response theories in the two channels. For a homogeneous system, the BCS Hamiltonian can be expressed as

$$
\begin{aligned}
H_{\mathrm{BCS}}= & \sum_{\mathbf{k} \sigma} \psi_{\mathbf{p} \sigma}^{\dagger} \xi_{\mathbf{p}} \psi_{\mathbf{p} \sigma}+\sum_{\mathbf{p}} \Delta_{\mathrm{Sc}} \psi_{-\mathbf{p} \uparrow} \psi_{\mathbf{p} \downarrow} \\
& +\sum_{\mathbf{p}} \Delta_{\mathrm{sc}} \psi_{\mathbf{p} \downarrow}^{\dagger} \psi_{-\mathbf{p} \uparrow}^{\dagger},
\end{aligned}
$$

where $\xi_{\mathbf{p}}=\mathbf{p}^{2} / 2 m-\mu$. As a familiar result, the BCS Green and anomalous Green functions in the momentum space are

$$
\begin{aligned}
& G_{\mathrm{sc}}\left(i \omega_{n}, \mathbf{p}\right)=\frac{u_{\mathrm{sc} \mathbf{p}}^{2}}{i \omega_{n}-E_{\mathrm{scp}}}+\frac{v_{\mathrm{sc} \mathbf{p}}^{2}}{i \omega_{n}+E_{\mathrm{sc} \mathbf{p}}}, \\
& F_{\mathrm{sc}}\left(i \omega_{n}, \mathbf{p}\right)=-\frac{u_{\mathrm{scp}} v_{\mathrm{sc} \mathbf{p}}}{i \omega_{n}-E_{\mathrm{sc} \mathbf{p}}}+\frac{u_{\mathrm{sc} p} v_{\mathrm{sc} p}}{i \omega_{n}+E_{\mathrm{sc} p}},
\end{aligned}
$$

where $i \omega_{n}$ is the Fermion Matsubara frequency and $E_{\text {scp }}=$ $\sqrt{\xi_{\mathbf{p}}^{2}+\Delta_{\mathrm{sc}}^{2}}$ is the quasiparticle energy dispersion. Hereinafter we use the subscript "sc" to emphasize that these discussions are only under the BCS mean field approximation. Define $P \equiv$ $\left(i \omega_{n}, \mathbf{p}\right)$; the number and gap equations are determined by $n=$ $2 \sum_{P} G_{\mathrm{sc}}(P)$ and $\Delta_{\mathrm{sc}}=-g \sum_{P} F_{\mathrm{sc}}(P)$. These identities give

$$
\begin{aligned}
& n=\sum_{\mathbf{p}}\left[1-\frac{\xi_{\mathrm{scp}}}{E_{\mathrm{scp}}}\left(1-2 f\left(E_{\mathrm{scp}}\right)\right)\right], \\
& \frac{1}{g}=\sum_{\mathbf{p}} \frac{1-2 f\left(E_{\mathrm{scp}}\right)}{2 E_{\mathrm{scp}}} .
\end{aligned}
$$

The bare Green function is $G_{0}(P)=\left(i \omega_{n}-\xi_{\mathbf{p}}\right)^{-1}$. The Dyson equation gives $G_{\mathrm{sc}}^{-1}(P)=G_{0}^{-1}(P)-\Sigma_{\mathrm{sc}}(P)$ where $\Sigma_{\mathrm{sc}}(P)=$ $-\Delta_{\text {sc }}^{2} G_{0}(-P)$ is the BCS self-energy.

2.1. Density Channel. In the density channel, the system is perturbed by an effective external EM field $A^{\mu}$, and the Hamiltonian becomes $H=H_{\mathrm{BCS}}+H_{I}$ with

$$
H_{I}=\sum_{\mathbf{p q} \sigma} \psi_{\mathbf{p}+\mathbf{q} \sigma}^{\dagger} \gamma^{\mu}(\mathbf{p}+\mathbf{q}, \mathbf{p}) A_{\mu \mathbf{q}} \psi_{\mathbf{p} \sigma}
$$

where $\gamma^{\mu}(\mathbf{p}+\mathbf{q}, \mathbf{p}) \equiv \gamma^{\mu}(P+Q, P)=(1,(\mathbf{p}+\mathbf{q} / 2) / m)$ is the bare EM interaction vertex. Here $Q \equiv q^{\mu}=\left(i \Omega_{l}, \mathbf{q}\right)$ is the external four momentum, where $\Omega_{l}$ is the boson Matsubara frequency. The bare vertex satisfies the "bare" Ward identity

$$
q_{\mu} \gamma^{\mu}(P+Q, P)=G_{0}^{-1}(P+Q)-G_{0}^{-1}(P) .
$$




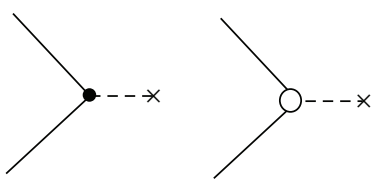

Figure 1: EM interaction vertex. The left one is the bare vertex, and the right one is the full vertex. The solid line denotes the fermion line and the dashed line denotes the photon line.

In a gauge invariant EM linear response theory, a full EM interaction vertex $\Gamma^{\mu}$ (the bare and full EM vertices are shown in Figure 1) which satisfies the full Ward identity

$$
q_{\mu} \Gamma_{\mathrm{sc}}^{\mu}(P+Q, P)=G_{\mathrm{sc}}^{-1}(P+Q)-G_{\mathrm{sc}}^{-1}(P)
$$

must be found so that the perturbed current can be expressed as $\delta J^{\mu}(Q)=K_{\mathrm{sc}}^{\mu \nu}(Q) A_{\nu}(\mathrm{Q})$ with $K_{\mathrm{sc}}^{\mu \nu}(Q)$ determined by the Kubo formalism

$$
\begin{aligned}
& K_{\mathrm{sc}}^{\mu \nu}(Q)=\frac{n}{m} h^{\mu \nu}+2 \sum_{P} \Gamma_{\mathrm{sc}}^{\mu}(P+Q, P) G_{\mathrm{sc}}(P+Q) \\
& \cdot \gamma^{\nu}(P, P+Q) G_{\mathrm{sc}}(P),
\end{aligned}
$$

where $h^{\mu \nu}=-\eta^{\mu \nu}\left(1-\eta^{\nu 0}\right)$ with $\eta^{\mu \nu}=\operatorname{diag}(1,-1,-1,-1)$ being the metric tensor. By using the Ward identity (8), it is easy to show that $q_{\mu} K_{\mathrm{sc}}^{\mu \nu}(Q)=0$, which further leads to the conservation of perturbed current $q_{\mu} \delta J^{\mu}(Q)=0$. Hence the linear response theory is indeed gauge invariant. Under the framework of BCS mean field theory, such full vertex can be obtained either by Nambu's integral-equation approach $[23,24]$ or by the CFOP approach. However, the Q-limit Ward identity provides an independent consistency check of the theory. It is not known if Nambu's approach can pass this check, while the vertex given by the latter approach is proved to satisfy this condition

$$
\left.\lim _{\mathbf{q} \rightarrow \mathbf{0}} \Gamma_{\mathrm{sc}}^{0}(P+Q, P)\right|_{\omega=0}=\frac{\partial G_{\mathrm{sc}}^{-1}(P)}{\partial \mu}=1-\frac{\partial \Sigma_{\mathrm{sc}}(P)}{\partial \mu} .
$$

Details can be found in [18]. This identity not only builds a consistent connection between the one-particle thermodynamics and two-particle response functions but also acts as the sufficient and necessary condition for the compressibility sum rule

$$
\frac{\partial n}{\partial \mu}=-K_{s c}^{00}(0, \mathbf{q} \longrightarrow \mathbf{0}) .
$$

The expression of this gauge invariant interaction vertex given by the CFOP approach is given in our past work [18]

$$
\begin{aligned}
\Gamma_{\mathrm{sc}}^{\mu}(P+Q, P)= & \gamma^{\mu}(P+Q, P)+\operatorname{Coll}_{\mathrm{sc}}^{\mu}(P+Q, P) \\
& +\operatorname{MT}_{\mathrm{sc}}^{\mu}(P+Q, P),
\end{aligned}
$$

where the second term,

$$
\begin{aligned}
\operatorname{Coll}_{s c}^{\mu}(P+Q, P)= & \Delta_{s c} \Pi^{\mu}(Q) G_{0}(-P-Q) \\
& +\Delta_{s c} \bar{\Pi}^{\mu}(Q) G_{0}(-P)
\end{aligned}
$$

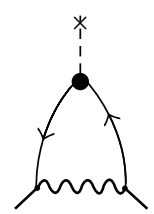

Figure 2: Maki-Thompson diagram. The wavy line represents the pair propagator.

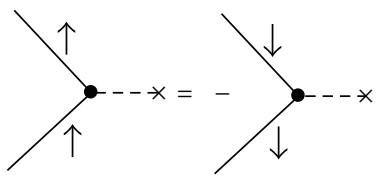

FIGURE 3: Spin interaction vertex. It has different signs for different pseudospin indices.

corresponds to the excitations of Nambu-Goldstone modes due to the breaking of the $U(1)$ symmetry and the third term,

$$
\begin{aligned}
& \mathrm{MT}_{\mathrm{sc}}^{\mu}(P+Q, P) \\
& \quad=-\Delta_{\mathrm{sc}}^{2} G_{0}(-P) \gamma^{\mu}(-P,-P-Q) G_{0}(-P-Q),
\end{aligned}
$$

is the famous MT diagram which is shown in Figure 2. The expressions of $\Pi^{\mu}$ and $\bar{\Pi}^{\mu}$ are given in Appendix A. These two terms originates from summing up the diagrams with photon-fermion interaction lines inserted at any possible position. By using the equalities [18] $q_{\mu} \Pi^{\mu}(Q)=2 \Delta_{\text {sc }}$ and $q_{\mu} \bar{\Pi}^{\mu}(Q)=-2 \Delta_{\text {sc }}$, it can be shown that

$$
q_{\mu} \operatorname{Coll}_{\mathrm{sc}}^{\mu}(P+Q, P)=2 \Sigma_{\mathrm{sc}}(P)-2 \Sigma_{\mathrm{sc}}(P+Q) .
$$

Combining with the equality

$$
q_{\mu} \mathrm{MT}_{\mathrm{sc}}^{\mu}(P+Q, P)=\Sigma_{\mathrm{sc}}(P+Q)-\Sigma_{\mathrm{sc}}(P),
$$

the Ward identity (8) in the BCS mean field level can be proved.

2.2. Spin Channel. In the spin channel, the $U(1)$ spin rotational symmetry is also "gauged" by introducing an effective external field. However, this symmetry is not broken by the order parameter. Therefore, we expect that the structure of the spin linear response theory is simpler than that of its density counterpart. The "bare" spin interaction vertex is dependent on the pseudospin: $\gamma_{S \sigma}^{\mu}(\mathbf{p}+\mathbf{q}, \mathbf{p}) \equiv \gamma_{S \sigma}^{\mu}(P+Q, P)=S_{\sigma}(1,(\mathbf{p}+$ $\mathbf{q} / 2) / m$ ) with $S_{\uparrow}=1, S_{\downarrow}=-1$ and the subscript " $S$ " referring to the "spin." The quantity $S_{\sigma}$ indicates that the vertex has different signs for different pseudospin indices; see Figure 3. It also respects the "bare" Ward identity in the spin channel

$$
q_{\mu} \gamma_{S \sigma}^{\mu}(P+Q, P)=S_{\sigma}\left(G_{0}^{-1}(P+Q)-G_{0}^{-1}(P)\right) .
$$

The spin interaction Hamiltonian is given by

$$
H_{I S}=\sum_{\mathbf{p q} \sigma} \psi_{\mathbf{p}+\mathbf{q} \sigma}^{\dagger} \gamma_{S \sigma}^{\mu}(\mathbf{p}+\mathbf{q}, \mathbf{p}) A_{\mu \mathbf{q}} \psi_{\mathbf{p} \sigma} .
$$


Similarly, the perturbed spin current is also evaluated via the Kubo formalism $\delta J_{S}^{\mu}(Q)=K_{S s c}^{\mu \nu}(Q) A_{\nu}(Q)$ where

$$
\begin{aligned}
& K_{S \mathrm{sc}}^{\mu \nu}(Q)=\frac{n}{m} h^{\mu \nu}+\sum_{P \sigma} \Gamma_{S \mathrm{sc} \sigma}^{\mu}(P+Q, P) G_{\mathrm{sc}}(P+Q) \\
& \cdot \gamma_{S \sigma}^{\nu}(P, P+Q) G_{\mathrm{sc}}(P)
\end{aligned}
$$

The full spin interaction vertex $\Gamma_{S s c \sigma}^{\mu}$ is given by

$$
\begin{aligned}
\Gamma_{S s c \sigma}^{\mu}(P+Q, P)= & \gamma_{S \sigma}^{\mu}(P+Q, P) \\
& +\operatorname{MT}_{S s c \sigma}^{\mu}(P+Q, P),
\end{aligned}
$$

where the MT term is expressed as

$$
\begin{aligned}
& \mathrm{MT}_{S \mathrm{~s} c \sigma}^{\mu}(P+Q, P) \\
& \quad=-\Delta_{\mathrm{sc}}^{2} G_{0}(-P) \gamma_{S \sigma}^{\mu}(-P,-P-Q) G_{0}(-P-Q) .
\end{aligned}
$$

Since the $U(1)$ spin rotational symmetry is not broken below $T_{c}$, then the full spin interaction vertex does not contain contributions associated with the Nambu-Goldstone modes. Moreover, the Ward identity is indeed satisfied in the mean field theory level

$$
q_{\mu} \Gamma_{S \mathrm{sc} \sigma}^{\mu}(P+Q, P)=S_{\sigma}\left(G_{\mathrm{sc}}^{-1}(P+Q)-G_{\mathrm{sc}}^{-1}(P)\right) .
$$

Therefore, the perturbed spin current is conserved $q_{\mu} \delta J_{S}^{\mu}(Q)=0$. In the spin channel, there is no well-defined Q-limit Ward identity for unpolarized Fermi superfluids although such identity does exist for polarized Fermi superfluids [19]. This is because the equal-population case can not be approached from the population imbalanced case by simply letting the particle number difference approach zero.

The above discussions show that the linear response theories in the density and spin channels are fully consistent with the BCS mean field approximation for Fermi superfluids. However, when generalized to the whole BCS-BEC crossover regime, the mean field approximation overestimates the critical temperature in the unitarity and BEC side since the fluctuations of the noncondensed pairs are ignored. We next show a formally theoretical scheme in which the fundamental constraints are still satisfied when the pairing fluctuation effects are included.

\section{Gauge Invariant Linear Response Theories in the $G_{0} G$ Formalism}

When we consider the situation that the interaction between fermions is stronger than the BCS attraction, the self-energy obtains corrections from the noncondensed pairs. Hence the interaction vertex must be corrected correspondingly to ensure an exact validity of the Ward identity and Q-limit Ward identity. In this paper, we adapt the $G_{0} G$ formalism [25], which is compatible with the BCS-Leggett ground state, to discuss the pseudogap effect. The self-energy due to the noncondensed pair is given by

$$
\Sigma_{\mathrm{pg}}(P)=\sum_{\mathrm{Q}} t_{\mathrm{pg}}(Q) G_{0}(Q-P)
$$

where $t_{\mathrm{pg}}(\mathrm{Q})=-g^{\prime} /\left(1-g^{\prime} \chi(Q)\right)$ is the $t$-matrix due to noncondensed pairs. The pair susceptibility is constructed in the $G_{0} G$ formalism $\chi(Q)=\sum_{K} G(K) G_{0}(Q-K)$, where $G$ is the full Green function with pairing fluctuation effect included. Here we assume that the coupling constant between fermions in noncondensed pairs, $g^{\prime}$, is not necessarily equal to $g$, the coupling constant between fermions in condensed pairs. To determine the pairing onset temperature $T^{*}$, we still use the Thouless criterion; that is, $t_{\mathrm{pg}}(0)$ is divergent at $T^{*}$ or

$$
t_{\mathrm{pg}}^{-1}(0)=1+g^{\prime} \chi(0)=0
$$

One possible reason that $g^{\prime}$ may not be equal to $g$ is that the Thouless criterion can not reduce to the BCS gap equation even when $g^{\prime}$ is equal to $g$. Similarly, the $t$-matrix due to the condensed pair is $t_{\mathrm{sc}}(Q)=-\left(\Delta_{\mathrm{sc}}^{2} / T\right) \delta(Q)$, and the BCS self-energy is also expressed as $\Sigma_{\mathrm{sc}}(P)=\sum_{\mathrm{Q}} t_{\mathrm{sc}}(\mathrm{Q}) G_{0}(\mathrm{Q}-$ $P)$. The order parameter is still determined by $\Delta_{\mathrm{sc}}=$ $-g \sum_{\mathbf{p}}\left\langle\psi_{\mathbf{p} \uparrow} \psi_{-\mathbf{p} \downarrow}\right\rangle$, which is nonzero below $T_{c}$. Now the full inverse Green function is given by

$$
\begin{aligned}
G^{-1}(P) & =G_{0}^{-1}(P)-\Sigma(P) \\
& =G_{0}^{-1}(P)-\Sigma_{\mathrm{sc}}(P)-\Sigma_{\mathrm{pg}}(P),
\end{aligned}
$$

where $\Sigma(P)$ is the total self-energy. We emphasize that no further approximation is introduced now. The full theory is quite complicated due to the inclusion of the undetermined pseudogap self-energy $\Sigma_{\mathrm{pg}}$ and new coupling $g^{\prime}$; this brings difficulties to the numerical work in the future. If we adapt the approximation that the pair propagator is highly peaked at zero momentum as discussed in [25], the numerical calculation will be greatly simplified. However, this is not the aim of this paper. We hope to find a theory which can pass all stringent consistent types of testing in the first step.

3.1. Density Channel. In the density channel, to get a new gauge invariant interaction vertex, we must find a vertex correction which is consistent with the new self-energy $\Sigma_{\mathrm{pg}}$. Such vertex does exist if we adapt the upper modified $G_{0} G$ formalism

$$
\begin{aligned}
\Gamma^{\mu}(P+Q, P)= & \gamma^{\mu}(P+Q, P)+\operatorname{Coll}_{\mathrm{sc}}^{\mu}(P+Q, P) \\
& +\operatorname{MT}_{\mathrm{sc}}^{\mu}(P+Q, P) \\
& +\mathrm{MT}_{\mathrm{pg}}^{\mu}(P+Q, P)+\mathrm{AL}_{1}^{\mu}(P+Q, P) \\
& +\mathrm{AL}_{2}^{\mu}(P+Q, P)
\end{aligned}
$$

Here $\mathrm{Coll}_{\mathrm{sc}}^{\mu}$ and $\mathrm{MT}_{\mathrm{sc}}^{\mu}$ are still given by (13) and (14), respectively, and $\mathrm{MT}_{\mathrm{sc}}^{\mu}$ can be further expressed in a more general style by including the $t$-matrix $t_{\mathrm{sc}}$ :

$$
\begin{aligned}
& \mathrm{MT}_{\mathrm{sc}}^{\mu}(P+Q, P)=\sum_{K} t_{\mathrm{sc}}(K) G_{0}(K-P) \\
& \cdot \gamma^{\mu}(K-P, K-P-Q) G_{0}(K-P-Q) .
\end{aligned}
$$




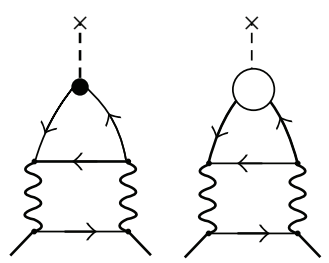

Figure 4: The left one is the $\mathrm{AL}_{1}$ diagram, and the right one is the $\mathrm{AL}_{2}$ diagram. The thin and thick lines denote the bare and full Green's functions, respectively.

Hence the MT diagram for noncondensed pairs is given by

$$
\begin{aligned}
& \mathrm{MT}_{\mathrm{pg}}^{\mu}(P+Q, P)=\sum_{K} t_{\mathrm{pg}}(K) G_{0}(K-P) \\
& \cdot \gamma^{\mu}(K-P, K-P-Q) G_{0}(K-P-Q) .
\end{aligned}
$$

The fifth and sixth terms are two different types of AL diagrams (shown in Figure 4),

$$
\begin{gathered}
\mathrm{AL}_{1}^{\mu}(P+Q, P)=-\sum_{K, L} t_{\mathrm{pg}}(K) t_{\mathrm{pg}}(K+Q) G_{0}(K-P) \\
\cdot G(K-L) G_{0}(L+Q) \gamma^{\mu}(L+Q, L) G_{0}(L), \\
\mathrm{AL}_{2}^{\mu}(P+Q, P)=-\sum_{K, L} t_{\mathrm{pg}}(K) t_{\mathrm{pg}}(K+Q) G_{0}(K-P) \\
\cdot G_{0}(K-L) G(L+Q) \Gamma^{\mu}(L+Q, L) G(L) .
\end{gathered}
$$

We see that $\mathrm{AL}_{2}^{\mu}$ contains a full vertex; hence expression (26) is in fact a series. In this scheme, the pseudogap effect does not enter into the terms related to the collective modes; then the Coll $\mathrm{sc}_{\mathrm{sc}}^{\mu}$ term vanishes above $T_{c}$; hence there are no NambuGoldstone modes excitations, which is consistent with the fact that the $U(1)$ EM symmetry is unbroken.

The full vertex (26) satisfies the Ward identity

$$
q_{\mu} \Gamma^{\mu}(P+Q, P)=G^{-1}(P+Q)-G^{-1}(P)
$$

and the gauge invariant response functions now can be expressed as

$$
\begin{aligned}
& K^{\mu \nu}(Q) \\
& =\frac{n}{m} h^{\mu \nu} \\
& \quad+2 \sum_{P} \Gamma^{\mu}(P+Q, P) G(P+Q) \gamma^{\nu}(P, P+Q) G(P) .
\end{aligned}
$$

To prove the Ward identity we need a lemma

$$
\begin{aligned}
q_{\mu} & {\left[\frac{1}{2} \mathrm{AL}_{1}^{\mu}(P+Q, P)+\frac{1}{2} \mathrm{AL}_{2}^{\mu}(P+Q, P)\right.} \\
& \left.+\mathrm{MT}_{\mathrm{pg}}^{\mu}(P+Q, P)\right]=0 .
\end{aligned}
$$

This proof of this lemma is outlined in Appendix B. Moreover, by applying the bare Ward identity (7), we can show that

$$
\begin{aligned}
q_{\mu} & \mathrm{MT}_{\mathrm{pg}}^{\mu}(P+Q, P) \\
& =\sum_{K} t_{\mathrm{pg}}(K)[G(K-P-Q)-G(K-P)] \\
& =\Sigma_{\mathrm{pg}}(P+Q)-\Sigma_{\mathrm{pg}}(P)
\end{aligned}
$$

Hence

$$
\begin{aligned}
q_{\mu} & {\left[\mathrm{AL}_{1}^{\mu}(P+Q, P)+\mathrm{AL}_{2}^{\mu}(P+Q, P)\right.} \\
& \left.+\mathrm{MT}_{\mathrm{pg}}^{\mu}(P+Q, P)\right]=\Sigma_{\mathrm{pg}}(P)-\Sigma_{\mathrm{pg}}(P+Q) .
\end{aligned}
$$

Finally, (7), (15), (16), and (34) lead to the Ward identity (30) self-consistently by assuming that $\operatorname{AL}_{2}^{\mu}(P+Q, P)$ has a full gauge invariant interaction vertex.

Moreover, the full vertex should also respect the Q-limit Ward identity

$$
\left.\lim _{\mathbf{q} \rightarrow \mathbf{0}} \Gamma^{0}(P+Q, P)\right|_{\omega=0}=\frac{\partial G^{-1}(P)}{\partial \mu}=1-\frac{\partial \Sigma(P)}{\partial \mu} .
$$

A brief derivation shows that this identity ensures the compressibility sum rule

$$
\begin{aligned}
\frac{\partial n}{\partial \mu} & =-2 \sum_{P} G^{2}(P) \frac{\partial G^{-1}(P)}{\partial \mu}=-\lim _{\mathbf{q} \rightarrow \mathbf{0}} \sum_{P} \Gamma^{0}(P+Q, P) \\
& \left.\cdot G(P+Q) \gamma^{0}(P, P+Q) G(P)\right|_{\omega=0} \\
& =-\left.\lim _{\mathbf{q} \rightarrow \mathbf{0}} K^{00}(Q)\right|_{\omega=0},
\end{aligned}
$$

where $n=2 \sum_{P} G(P)$ now. Note that

$$
\begin{aligned}
\Gamma^{\mu}(P+Q, P)= & \Gamma_{\mathrm{sc}}^{\mu}(P+Q, P)+\mathrm{MT}_{\mathrm{pg}}^{\mu}(P+Q, P) \\
& +\mathrm{AL}_{1}^{\mu}(P+Q, P)+\mathrm{AL}_{2}^{\mu}(P+Q, P)
\end{aligned}
$$

and that $\Gamma_{\mathrm{sc}}^{\mu}(P+Q, P)$ already satisfies $(10)$; we only need to show that

$$
\begin{array}{r}
\lim _{\mathbf{q} \rightarrow \mathbf{0}}\left[\mathrm{MT}_{\mathrm{pg}}^{0}(P+Q, P)+\mathrm{AL}_{1}^{0}(P+Q, P)\right. \\
\left.+\mathrm{AL}_{2}^{0}(P+Q, P)\right]\left.\right|_{\omega=0}=-\frac{\partial \Sigma_{\mathrm{pg}}(P)}{\partial \mu} .
\end{array}
$$


It can be proved as follows:

$$
\begin{aligned}
- & \frac{\partial \sum_{\mathrm{pg}}(P)}{\partial \mu}=-\sum_{K} t_{\mathrm{pg}}(K) \frac{\partial G_{0}(K-P)}{\partial \mu} \\
& -\sum_{K} \frac{\partial t_{\mathrm{pg}}(K)}{\partial \mu} G_{0}(K-P)=\sum_{K} t_{\mathrm{pg}}(K) G_{0}^{2}(K-P) \\
& \cdot \frac{\partial G_{0}^{-1}(K-P)}{\partial \mu}+\sum_{K} t_{\mathrm{pg}}^{2}(K) \frac{\partial t_{\mathrm{pg}}^{-1}(K)}{\partial \mu} G_{0}(K-P) \\
& =\sum_{K} t_{\mathrm{pg}}(K) G_{0}^{2}(K-P)+\sum_{K} t_{\mathrm{pg}}^{2}(K) \\
& +\frac{\partial \chi_{\mathrm{pg}}(K)}{\partial \mu} G_{0}(K-P)=\sum_{K} t_{\mathrm{pg}}(K) G_{0}^{2}(K-P) \\
& +\sum_{K, L} t_{\mathrm{pg}}^{2}(K) \frac{\partial G_{0}(K-L)}{\partial \mu} G_{(L)} G_{0}(K-P) \\
& +\sum_{K, L} t_{\mathrm{pg}}^{2}(K) G_{0}(K-L) \frac{\partial G(L)}{\partial \mu} G_{0}(K-P) \\
& +\sum_{K} t_{\mathrm{pg}}(K) G_{0}^{2}(K-P)-\sum_{K, L} t_{\mathrm{pg}}^{2}(K) G_{0}^{2}(K-L) \\
& \cdot G^{-1}(L) G_{0}(K-P)-\sum_{\mathrm{pg}}^{2}(K) G_{0}(K-L) G^{2}(L) \\
& \frac{\partial \mu}{G_{0}}(K-P) .
\end{aligned}
$$

Since the 0 -component of the bare vertex $\gamma^{\mu}$ is always 1 , then right-hand side of (39) is indeed

$$
\mathrm{MT}_{\mathrm{pg}}^{0}(P, P)+\mathrm{AL}_{1}^{0}(P, P)+\mathrm{AL}_{2}^{0}(P, P)
$$

if we compare with expressions (28) and (29). Therefore, the Q-limit Ward identity is also satisfied.

3.2. Spin Channel. In the spin channel, the linear response theory is formulated in a similar but simpler way. The central idea is to find the gauge invariant spin interaction vertex. According to the expression of the spin interaction Hamiltonian (18), it is convenient to restore the pseudospin dependence of the Green function. Hence $G_{0 \uparrow}(P)=G_{0 \downarrow}(P)=$ $G_{0}(P), G_{\uparrow}(P)=G_{\downarrow}(P)=G(P)$. Generically, the vertex also contains the MT and AL diagrams when going beyond BCS theory:

$$
\begin{aligned}
\Gamma_{S \sigma}^{\mu}(P+Q, P)= & \gamma_{S \sigma}^{\mu}(P+Q, P)+\mathrm{MT}_{S s c \sigma}^{\mu}(P+Q, P) \\
& +\mathrm{MT}_{S \mathrm{pg} \sigma}^{\mu}(P+Q, P) \\
& +\mathrm{AL}_{S 1 \sigma}^{\mu}(P+Q, P) \\
& +\mathrm{AL}_{S 2 \sigma}^{\mu}(P+Q, P) .
\end{aligned}
$$

The MT diagram associated with the contributions from the order parameter and pseudogap are, respectively, given by

$$
\begin{gathered}
\mathrm{MT}_{S \mathrm{sc} \sigma}^{\mu}(P+Q, P)=\sum_{K} t_{\mathrm{sc}}(K) G_{0 \bar{\sigma}}(K-P) \\
\cdot \gamma_{S \bar{\sigma}}^{\mu}(K-P, K-P-Q) G_{0 \bar{\sigma}}(K-P-Q), \\
\mathrm{MT}_{S \mathrm{pg} \sigma}^{\mu}(P+Q, P)=\sum_{K} t_{\mathrm{pg}}(K) G_{0 \bar{\sigma}}(K-P) \\
\cdot \gamma_{S \bar{\sigma}}^{\mu}(K-P, K-P-Q) G_{0 \bar{\sigma}}(K-P-Q) .
\end{gathered}
$$

Here we emphasize again that the spin interaction vertices have different signs for different pseudospin indices, which leads to an important result that the contributions from the AL diagrams automatically cancel out. This can be shown by a straightforward verification. Figure 5 shows the vertex correction from the two spin-up $\mathrm{AL}_{1}$ diagrams (with two sets of different pseudospin attributions). We have

$$
\begin{aligned}
& \mathrm{AL}_{S 1 \uparrow}^{\mu}(P+Q, P)=-\sum_{K, K^{\prime}} G_{0 \downarrow}(K-P) t_{\mathrm{pg}}(K+Q) \\
& \cdot t_{\mathrm{pg}}\left(K^{\prime}\right)\left[G_{\uparrow}\left(K-K^{\prime}\right) G_{0 \downarrow}\left(K^{\prime}+Q\right)\right. \\
& \cdot \gamma_{S \downarrow}^{\mu}\left(K^{\prime}+Q, K^{\prime}\right) G_{0 \downarrow}\left(K^{\prime}\right)+G_{\downarrow}\left(K-K^{\prime}\right) \\
& \left.\cdot G_{0 \uparrow}\left(K^{\prime}+Q\right) \gamma_{S \uparrow}^{\mu}\left(K^{\prime}+Q, K^{\prime}\right) G_{0 \uparrow}\left(K^{\prime}\right)\right]=0,
\end{aligned}
$$

where the fact that $G_{0 \uparrow}=G_{0 \downarrow}, G_{\uparrow}=G_{\downarrow}$, and $\gamma_{S \uparrow}^{\mu}=$ $-\gamma_{S \downarrow}^{\mu}$ has been applied. Similar calculation indicates that the vertex corrections from the two spin-down $\mathrm{AL}_{1}$ diagrams also vanish. The vertex corrections from spin-up $\mathrm{AL}_{2}$ are

$$
\begin{gathered}
\mathrm{AL}_{S 2 \uparrow}^{\mu}(P+Q, P)=-\sum_{K, K^{\prime}} G_{0 \downarrow}(K-P) t_{\mathrm{pg}}(K+Q) \\
\cdot t_{\mathrm{pg}}\left(K^{\prime}\right)\left[G_{0 \uparrow}\left(K-K^{\prime}\right) G_{\downarrow}\left(K^{\prime}+Q\right)\right. \\
\cdot \Gamma_{S \downarrow}^{\mu}\left(K^{\prime}+Q, K^{\prime}\right) G_{\downarrow}\left(K^{\prime}\right)+G_{0 \downarrow}\left(K-K^{\prime}\right) \\
\left.\cdot G_{\uparrow}\left(K^{\prime}+Q\right) \Gamma_{S \uparrow}^{\mu}\left(K^{\prime}+Q, K^{\prime}\right) G_{\uparrow}\left(K^{\prime}\right)\right]=0 .
\end{gathered}
$$

Hence the contributions from two spin-down $\mathrm{AL}_{2}$ vanish too. From the equalities

$$
\begin{aligned}
& q_{\mu} \mathrm{MT}_{S \mathrm{sc} \sigma}^{\mu}(P+Q, P)=S_{\sigma}\left[\Sigma_{\mathrm{sc}}(P+Q)-\Sigma_{\mathrm{sc}}(P)\right] \\
& q_{\mu} \mathrm{MT}_{S \mathrm{pg} \sigma}^{\mu}(P+Q, P)=S_{\sigma}\left[\Sigma_{\mathrm{pg}}(P+Q)-\Sigma_{\mathrm{pg}}(P)\right]
\end{aligned}
$$

one can show that the Ward identity for the full spin interaction vertex is satisfied:

$$
q_{\mu} \Gamma_{S \sigma}^{\mu}(P+Q, P)=S_{\sigma}\left[G^{-1}(P+Q)-G^{-1}(P)\right] .
$$

The spin linear response theory is gauge invariant too.

Therefore, all consistency constraints are satisfied within this scheme in both the density and the spin channels when pairing fluctuation effects are considered. However, 


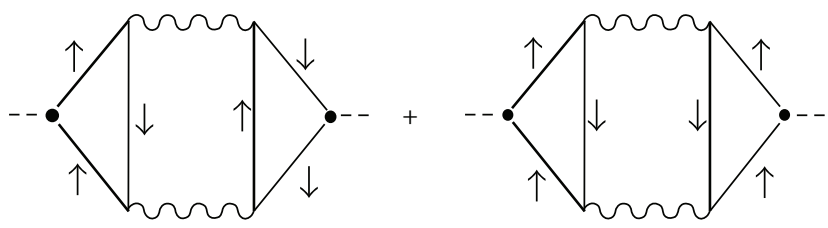

Figure 5: Vertex corrections from spin-up $\mathrm{AL}_{1}$ diagrams.

the approach in the density channel is not a useful form for numerical application. If any approximation is applied, it may most possibly violate some of the constraints. In a certain situation, some constraint may survive; hence these consistency conditions can play an indicator to "measure" how good the approximation is.

3.3. Applications. After formulating the theoretical framework, it is possible to evaluate some physical quantities under some conditions. As we have pointed out previously, if certain approximations are introduced, the fundamental constraints may not be satisfied. Our ideal future goal is to control the badness that those constraints are broken, which seems terribly difficult till now. For an instructive discussion, here we show how to derive physical quantities by applying certain approximation. We assume that the pair propagator is highly peaked at zero momentum [25]; then the pseudogap selfenergy can be approximated as

$$
\Sigma_{\mathrm{pg}}(-P) \simeq\left(\sum_{\mathrm{Q}} t_{\mathrm{pg}}(Q)\right) G_{0}(-P)=-\Delta_{\mathrm{pg}}^{2} G_{0}(-P),
$$

where $\Delta_{\mathrm{pg}}^{2}=-\sum_{\mathrm{Q}} t_{\mathrm{pg}}(\mathrm{Q})$ is the pseudogap, which is still hard to determine by numerics. If we define the total energy gap $\Delta^{2}=\Delta_{\mathrm{sc}}^{2}+\Delta_{\mathrm{pg}}^{2}$, then full Green's function given, $G(P)$, formally has a similar expression as BCS Green's function, $G_{\mathrm{sc}}(P)$, only with $\Delta_{\mathrm{sc}}$ replaced by $\Delta$. The MT diagram for noncondensed pairs can also be simplified as

$$
\begin{aligned}
& \mathrm{MT}_{\mathrm{pg}}^{\mu}(P+Q, P) \\
& \quad=-\Delta_{\mathrm{pg}}^{2} G_{0}(-P) \gamma^{\mu}(-P,-P-Q) G_{0}(-P-Q) .
\end{aligned}
$$

Using these results and applying Lemma (32) we can get the expression for the response kernel $K^{\mu \nu}(Q)$, from which we further derive an approximated expression of the compressibility. Consider

$$
\begin{aligned}
\frac{\partial n}{\partial \mu}= & \sum_{\mathbf{p}}\left[\frac{\Delta_{\text {sc }}^{2}}{E_{\mathbf{p}}^{3}}\left(1-2 f\left(E_{\mathbf{p}}\right)\right)-2\left(1-\frac{\Delta_{\mathrm{pg}}^{2}}{E_{\mathbf{p}}^{2}}\right) \frac{\xi_{\mathbf{p}}^{2}}{E_{\mathbf{p}}^{2}} \frac{\partial f\left(E_{\mathbf{p}}\right)}{\partial E_{\mathbf{p}}}\right] \\
& +\frac{\left[\sum_{\mathbf{p}}\left(\xi_{\mathbf{p}} / E_{\mathrm{scp}}^{2}\right)\left(\left(1-2 f\left(E_{\mathrm{scp}}\right)\right) / E_{\mathrm{scp}}+2\left(\partial f\left(E_{\mathrm{scp}}\right) / \partial E_{\mathrm{scp}}\right)\right)\right]^{2}}{\sum_{\mathbf{p}}\left(1 / E_{\mathrm{scp}}\right)\left(\left(1-2 f\left(E_{\mathrm{scp}}\right)\right) / E_{\mathrm{scp}}+2\left(\partial f\left(E_{\mathrm{scp}}\right) / \partial E_{\mathrm{scp}}\right)\right)},
\end{aligned}
$$

where $E_{\mathbf{p}}=\sqrt{\xi_{\mathbf{p}}^{2}+\Delta^{2}}$. The second term comes from the breaking of the $U(1)$ symmetry in the superfluid phase; it vanishes above $T_{c}$ since the symmetry is unbroken in the normal phase. This approximation obviously breaks the Ward identity, and we hope to find a way to control the approximation in the future.

\section{Conclusion}

We have constructed gauge invariant density and spin linear response theories for a Fermi gas undergoing BCS-BEC crossover by including adequate diagrams in the interaction vertices using the $t$-matrix formalism based on a slightly corrected $G_{0} G$ scheme. We verified that the Ward identities and the important Q-limit Ward identity are both satisfied when the contributions due to the order parameter (condensed pairs) and pseudogap (noncondensed pairs) are both included. This justifies Nambu's assertion that the modification of the vertex must be consistent with the way that the self-energy is included in the quasiparticle. Those constraints guarantee the self-consistency of the theories. Until now our approach is a purely theoretical formalism without including any approximation, yet we believe it will shed light on the reliable theoretical predictions of the transport properties of strongly correlated Fermi gases and help us to understand more about the many particle theory. Future improvements include trustworthy numerical calculations by taking suitable approximations.

\section{Appendices}

\section{A. Vertex Correction in BCS Mean Field Theory}

The BCS full vertices are given by:

$$
\begin{gathered}
\Pi^{\mu}=\frac{Q_{1} Q_{4}^{\mu}-Q_{3} Q_{5}^{\mu}}{Q_{1} Q_{2}-Q_{3}^{2}}, \\
\bar{\Pi}^{\mu}=\frac{Q_{2} Q_{5}^{\mu}-Q_{3} Q_{4}^{\mu}}{Q_{1} Q_{2}-Q_{3}^{2}} .
\end{gathered}
$$

Here

$$
\begin{aligned}
& Q_{1}(Q)=\frac{1}{g}+\sum_{P} G_{s c}(P-Q) G_{s c}(-P), \\
& Q_{2}(Q)=\frac{1}{g}+\sum_{P} G_{s c}(P+Q) G_{s c}(-P),
\end{aligned}
$$




$$
\begin{aligned}
& Q_{3}(Q)=-\sum_{P} F_{\mathrm{sc}}(P+Q) F_{\mathrm{sc}}(P), \\
& Q_{4}^{\mu}(Q)=-2 \sum_{P} \gamma^{\mu}(P+Q, P) G_{\mathrm{sc}}(P+Q) F_{\mathrm{sc}}(P), \\
& Q_{5}^{\mu}(Q)=-2 \sum_{P} \gamma^{\mu}(P+Q, P) F_{\mathrm{sc}}(P+Q) G_{\mathrm{sc}}(P) .
\end{aligned}
$$

\section{B. Proof of the Lemma}

Since $\Sigma_{\mathrm{pg}}(P+Q)=\sum_{K} t_{\mathrm{pg}}(K) G_{0}(K-P-Q)=\sum_{K} t_{\mathrm{pg}}(K+$ Q) $G_{0}(K-P)$, therefore

$$
\begin{aligned}
0= & \sum_{K}\left[t_{\mathrm{pg}}(K+Q) G_{0}(K-P)-t_{\mathrm{pg}}(K)\right. \\
& \left.\cdot G_{0}(K-P-Q)\right]=\sum_{K}\left(\left[t_{\mathrm{pg}}(K+Q)-t_{\mathrm{pg}}(K)\right]\right. \\
& \cdot G_{0}(K-P)+t_{\mathrm{pg}}(K) \\
& \left.\cdot\left[G_{0}(K-P)-G_{0}(K-P-Q)\right]\right)=\sum_{K}\left(t_{\mathrm{pg}}(K)\right. \\
& \cdot\left[G_{0}(K-P)-G_{0}(K-P-Q)\right]-t_{\mathrm{pg}}(K+Q) \\
& \left.\cdot t_{\mathrm{pg}}(K)[\chi(K+Q)-\chi(K)] G_{0}(K-P)\right) .
\end{aligned}
$$

Note that $\chi(K)=\sum_{L} G(K-L) G_{0}(L)=\sum_{L} G_{0}(K-L) G(L)$; we have

$$
\begin{aligned}
& \chi(K+Q)-\chi(K)=\frac{1}{2} \\
& \quad \cdot \sum_{L}\left(G(K-L)\left[G_{0}(L+Q)-G_{0}(L)\right]\right. \\
& \left.\quad+G_{0}(K-L)[G(L+Q)-G(L)]\right) .
\end{aligned}
$$

Using this equality, we get

$$
\begin{aligned}
0= & -\frac{1}{2} \sum_{K L} t_{\mathrm{pg}}(K+Q) t_{\mathrm{pg}}(K) G_{0}(K-P) \\
& \cdot\left(G(K-L)\left[G_{0}(L+Q)-G_{0}(L)\right]\right. \\
& \left.+G_{0}(K-L)[G(L+Q)-G(L)]\right)+\sum_{K} t_{\mathrm{pg}}(K) \\
& \cdot\left[G_{0}(K-P)-G_{0}(K-P-Q)\right] .
\end{aligned}
$$

By applying the bare WI (7) and WI (30), we can see that (B.3) leads to the lemma.

\section{Conflict of Interests}

There is no conflict of interests.

\section{Acknowledgments}

Hao Guo thanks the support by National Natural Science Foundation of China (Grant no. 11204032) and Natural Science Foundation of Jiangsu Province, China (SBK201241926).

\section{References}

[1] P. I. Arseev, S. O. Loiko, and N. K. Fedorov, "Theory of gauge-invariant response of superconductors to an external electromagnetic field," Physics-Uspekhi, vol. 49, no. 1, pp. 1-18, 2006.

[2] R. Combescot, M. Y. Kagan, and S. Stringari, "Collective mode of homogeneous superfluid Fermi gases in the BEC-BCS crossover," Physical Review A, vol. 74, Article ID 042717, 2006.

[3] T. Enss and R. Haussmann, "Quantum mechanical limitations to spin diffusion in the unitary Fermi gas," Physical Review Letters, vol. 109, Article ID 195303, 2012.

[4] F. Palestini, P. Pieri, and G. C. Strinati, "Density and spin response of a strongly interacting fermi gas in the attractive and quasirepulsive regime," Physical Review Letters, vol. 108, Article ID 080401, 2012.

[5] H. Guo, C. C. Chien, and K. Levin, "Establishing the presence of coherence in atomic fermi superfluids: spin-flip and spinpreserving Bragg scattering at finite temperatures," Physical Review Letters, vol. 105, Article ID 120401, 2010.

[6] H. Hu, X. J. Liu, and P. D. Drummond, "Static structure factor of a strongly correlated Fermi gas at large momenta," Europhysics Letters, vol. 91, no. 2, Article ID 20005, 2010.

[7] H. Hu, X. J. Liu, and P. D. Drummond, "Dynamic response of strongly correlated Fermi gases in the quantum virial expansion," Physical Review A, vol. 81, Article ID 033630, 2010.

[8] H. Hu and X. J. Liu, "Universal dynamic structure factor of a strongly correlated Fermi gas," Physical Review A, vol. 85, Article ID 023612, 2012.

[9] X. J. Liu and H. Hu, "Virial expansion for a strongly correlated Fermi gas with imbalanced spin populations," Physical Review A, vol. 82, no. 4, Article ID 043626, 7 pages, 2010.

[10] K. Seo and C. A. R. S. de Melo, "Compressibility and spin susceptibility in the evolution from BCS to BEC superfluids," http://arxiv.org/abs/1105.4365.

[11] Y. He and K. Levin, "Establishing conservation laws in paircorrelated many-body theories: T-matrix approaches," Physical Review B, vol. 89, Article ID 035106, 2014.

[12] C. Sanner, E. J. Su, A. Kesher et al., "Speckle imaging of spin fluctuations in a strongly interacting Fermi gas," Physical Review Letters, vol. 106, Article ID 010402, 2011.

[13] A. Sommer, M. Ku, G. Roati, and M. W. Zwierlein, "Universal spin transport in a strongly interacting Fermi gas," Nature, vol. 472, no. 7342, pp. 201-204, 2011.

[14] H. Hu, X. J. Liu, P. Dyke et al., "Universal behavior of pair correlations in a strongly interacting Fermi gas," Physical Review Letters, vol. 105, no. 7, Article ID 070402, 4 pages, 2010.

[15] S. Hoinka, M. Lingham, M. Delehaye, and C. J. Vale, "Dynamic spin response of a strongly interacting fermi gas," Physical Review Letters, vol. 109, no. 5, Article ID 050403, 2012.

[16] M. J. H. Ku, A. T. Sommer, L. W. Cheuk, and M. W. Zwierlein, "Revealing the superfluid lambda transition in the universal thermodynamics of a unitary fermi gas," Science, vol. 335, no. 6068, pp. 563-567, 2012.

[17] M. G. Lingham, K. Fenech, S. Hoinka, and C. J. Vale, "Local observation of pair condensation in a Fermi gas at unitarity," Physical Review Letters, vol. 112, no. 10, Article ID 100404, 2014.

[18] H. Guo, C.-C. Chien, and Y. He, "Theories of linear response in BCS superfluids and how they meet fundamental constraints," Journal of Low Temperature Physics, vol. 172, no. 1-2, pp. 5-46, 2013. 
[19] H. Guo, Y. Li, Y. He, and C. Chien, "Density and spin linear response of atomic Fermi superfluids with population imbalance in the BCS-BEC crossover," Journal of Physics B: Atomic, Molecular and Optical Physics, vol. 47, no. 8, Article ID 085302, 2014.

[20] H. Guo, C. C. Chien, Y. He, and K. Levin, "Fundamental constraints on linear response theories of Fermi superfluids above and below $\mathrm{T}_{c}$," International Journal of Modern Physics B, vol. 27, Article ID 1330010, 2013.

[21] G. D. Mahan, Many-Particle Physics, Kluwer Academic Publishers, Plenum Publishers, New York, NY, USA, 3rd edition, 2000.

[22] K. Yoshimi, T. Kato, and H. Maebashi, "Enhanced spin susceptibility toward the charge-ordering transition in a twodimensional extended hubbard model," Journal of the Physical Society of Japan, vol. 78, Article ID 104002, 11 pages, 2009.

[23] Y. Nambu, "Quasi-particles and gauge invariance in the theory of superconductivity," Physical Review, vol. 117, no. 3, pp. 648663, 1960.

[24] J. R. Schrieffer, Theory of Superconductivity, Benjamin, New York, NY, USA, 1964.

[25] Q. J. Chen, Y. He, C. C. Chien, and K. Levin, "Theory of radio frequency spectroscopy experiments in ultracold Fermi gases and their relation to photoemission in the cuprates," Reports on Progress in Physics, vol. 72, no. 12, Article ID 122501, 2009. 

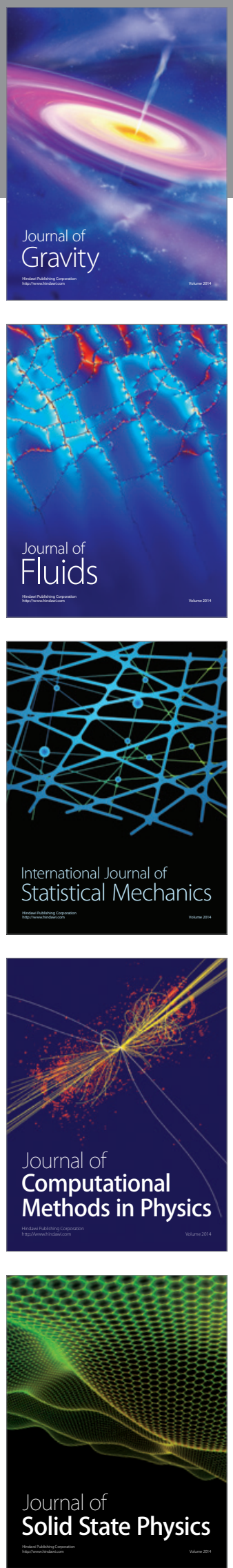

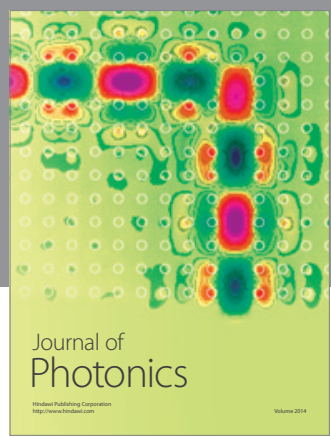

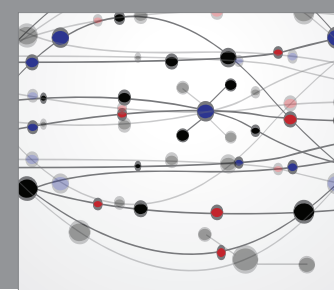

The Scientific World Journal

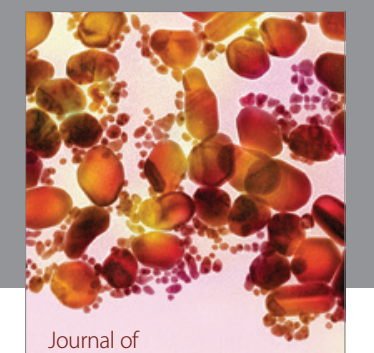

Soft Matter
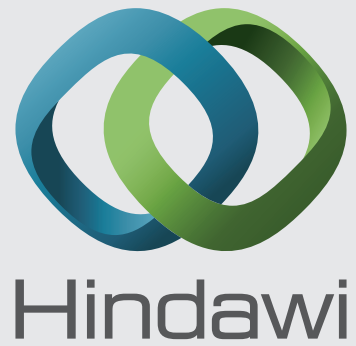

Submit your manuscripts at

http://www.hindawi.com
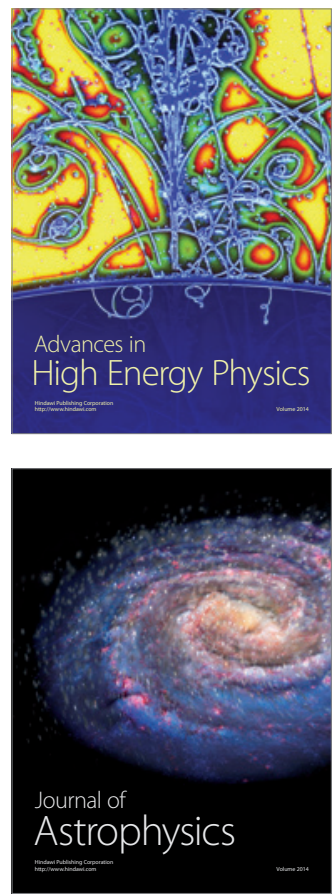
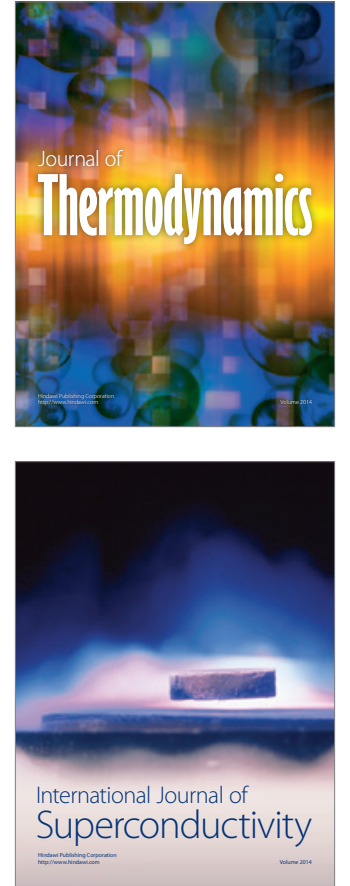
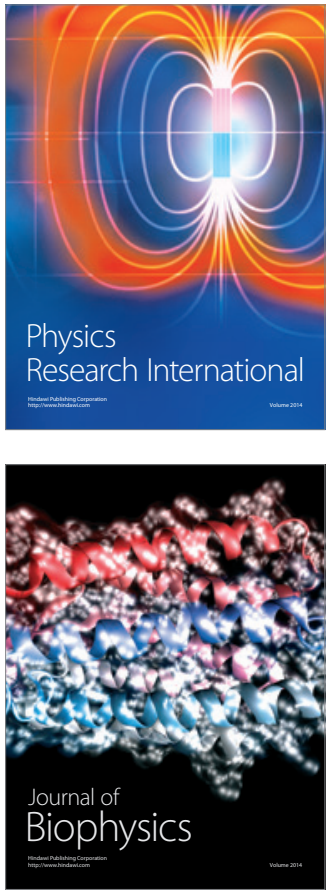
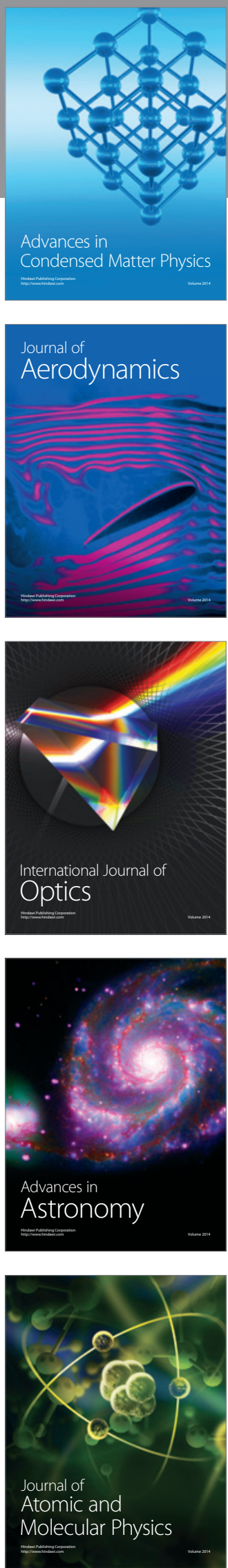